\title{
PROPERTIES OF AN ECLIPSING DOUBLE WHITE DWARF BINARY NLTT 11748
}

\author{
David L. Kaplan ${ }^{1}$, Thomas R. Marsh ${ }^{2}$, Arielle N. Walker ${ }^{1}$, Lars Bildsten ${ }^{3,4}$, \\ Madelon C. P. Bours ${ }^{2}$, Elmé Breedt ${ }^{2}$, Chris M. Copperwheat ${ }^{5}$, Vik S. Dhillon ${ }^{6}$, Steve B. Howell ${ }^{7}$, \\ Stuart P. LitTlefair ${ }^{6}$, Avi ShPORER ${ }^{8}$, and Justin D. R. SteinfadT ${ }^{4}$ \\ ${ }^{1}$ Physics Department, University of Wisconsin-Milwaukee, Milwaukee, WI 53211, USA; kaplan@uwm.edu. \\ ${ }^{2}$ Department of Physics, University of Warwick, Coventry CV4 7AL, UK \\ ${ }^{3}$ Kavli Institute for Theoretical Physics and Department of Physics, Kohn Hall, University of California, Santa Barbara, CA 93106, USA \\ ${ }^{4}$ Department of Physics, Broida Hall, University of California, Santa Barbara, CA 93106, USA \\ ${ }^{5}$ Astrophysics Research Institute, Liverpool John Moores University, IC2, Liverpool Science Park, 146 Brownlow Hill, Liverpool L3 5RF, UK \\ ${ }^{6}$ Department of Physics and Astronomy, University of Sheffield, Sheffield S3 7RH, UK \\ ${ }^{7}$ NASA Ames Research Center, Moffett Field, CA 94035, USA \\ ${ }^{8}$ California Institute of Technology, 1200 East California Boulevard, Pasadena, CA 91125, USA \\ Received 2013 July 19; accepted 2013 November 21; published 2013 December 20
}

\begin{abstract}
We present high-quality ULTRACAM photometry of the eclipsing detached double white dwarf binary NLTT 11748. This system consists of a carbon/oxygen white dwarf and an extremely low mass $\left(<0.2 M_{\odot}\right)$ helium-core white dwarf in a $5.6 \mathrm{hr}$ orbit. To date, such extremely low-mass white dwarfs, which can have thin, stably burning outer layers, have been modeled via poorly constrained atmosphere and cooling calculations where uncertainties in the detailed structure can strongly influence the eventual fates of these systems when mass transfer begins. With precise (individual precision $\approx 1 \%$ ), high-cadence $(\approx 2 \mathrm{~s})$, multicolor photometry of multiple primary and secondary eclipses spanning $>1.5 \mathrm{yr}$, we constrain the masses and radii of both objects in the NLTT 11748 system to a statistical uncertainty of a few percent. However, we find that overall uncertainty in the thickness of the envelope of the secondary carbon/oxygen white dwarf leads to a larger $(\approx 13 \%)$ systematic uncertainty in the primary He WD's mass. Over the full range of possible envelope thicknesses, we find that our primary mass $\left(0.136-0.162 M_{\odot}\right)$ and surface gravity $\left(\log (g)=6.32-6.38\right.$; radii are $\left.0.0423-0.0433 R_{\odot}\right)$ constraints do not agree with previous spectroscopic determinations. We use precise eclipse timing to detect the Rømer delay at $7 \sigma$ significance, providing an additional weak constraint on the masses and limiting the eccentricity to $e \cos \omega=(-4 \pm 5) \times 10^{-5}$. Finally, we use multicolor data to constrain the secondary's effective temperature $(7600 \pm 120 \mathrm{~K})$ and cooling age (1.6-1.7 Gyr).
\end{abstract}

Key words: binaries: eclipsing - stars: individual (NLTT 11748) - techniques: photometric - white dwarfs

Online-only material: color figures

\section{INTRODUCTION}

Among the more interesting products of binary evolution are compact binaries (periods less than 1 day) containing heliumcore white dwarfs (WDs). These WDs are created from lowmass $\left(<2.0 M_{\odot}\right)$ stars when stellar evolution is truncated by a binary companion before the He core reaches the $\approx 0.48 M_{\odot}$ needed for the helium core flash. Such WDs were first identified both as companions to millisecond pulsars (Lorimer et al. 1995; van Kerkwijk et al. 2005; Bassa et al. 2006) and other WDs (e.g., Bergeron et al. 1992; Marsh et al. 1995), with large numbers of double WD binaries discovered in recent years. In particular, the Extremely Low Mass (ELM) survey (Kilic et al. 2012; Brown et al. 2013, and references therein) has discovered tens of new He-core WDs in the last few years, focusing on the objects with masses $<0.2 M_{\odot}$.

The compact binaries containing these WDs will inspiral due to emission of gravitational radiation in less than a Hubble time; the most compact of them will merge in $<1 \mathrm{Myr}$ (Brown et al. 2011). When mass transfer begins, detailed evolutionary and mass transfer calculations (Marsh et al. 2004; D'Antona et al. 2006; Kaplan et al. 2012) will determine whether the objects remain separate (typically resulting in an AM CVn binary) or merge (as a R CrB star or possibly a Type Ia supernova; Iben \& Tutukov 1984; Webbink 1984). Essential to determining the fates of these systems (and hence making predictions for lowfrequency gravitational radiation and other end products) is an accurate knowledge of their present properties: their masses determine the in-spiral time and their radii and degrees of degeneracy help determine the stability of mass transfer (Deloye et al. 2005; D'Antona et al. 2006; Kaplan et al. 2012). This is particularly interesting for the ELM WDs, as they are predicted to possess stably burning $\mathrm{H}$ envelopes (with $\sim 10^{-3}-10^{-2} M_{\odot}$ of hydrogen) that keep them bright for Gyr (Serenelli et al. 2002; Panei et al. 2007) and increase their radii compared with "cold," fully degenerate WDs by a factor of two or more. This burning slows the cooling behavior of these objects (it may not be monotonic for all objects) and improving our understanding of ELM WD cooling would aid in evolutionary models for millisecond pulsars and later stages of mass transfer (e.g., Tauris et al. 2012; Antoniadis et al. 2012; Kaplan et al. 2013).

Few ELM WDs have had mass and radius measurements of any precision. As most systems are single-line spectroscopic binaries (Kaplan et al. 2012), precise masses are difficult to obtain (although some pulsar systems are better; Bassa et al. 2006; Antoniadis et al. 2012). Radii are even harder, typically relying on poorly calibrated surface gravity measurements and cooling models (as in Kilic et al. 2012). The eclipsing double WD binary NLTT 11748 (Steinfadt et al. 2010) allowed for the first geometric measurement of the radius of an ELM WD in the field (cf. PSR J1911-5958A in the globular cluster NGC 6752; Bassa et al. 2006), finding $R \approx 0.04 R_{\odot}$ for the $\approx 0.15 M_{\odot} \mathrm{He}$ WD, with new eclipsing systems (Parsons et al. 2011; Brown et al. 2011; Vennes et al. 2011) helping even more. However, 
Table 1

Log of ULTRACAM Observations and Eclipse Times

\begin{tabular}{|c|c|c|c|c|c|c|c|}
\hline Date & $\begin{array}{l}\text { Eclipse Time } \\
\text { (MBJD) }\end{array}$ & Telescope & Eclipse & Filters $^{\mathrm{a}}$ & $\begin{array}{c}\text { Exposures }^{\mathrm{a}} \\
\text { (s) }\end{array}$ & Num. Stars ${ }^{a}$ & $\begin{array}{c}\text { Precisions }^{\mathrm{a}} \\
(\%)\end{array}$ \\
\hline 2010 Nov $12 \ldots$ & $55512.182179(17)$ & NTT & secondary & $u^{\prime} g^{\prime} i^{\prime}$ & $7.69,2.55,2.55$ & $4,5,3$ & $2.9,1.0,1.3$ \\
\hline 2010 Nov $15 \ldots$ & $55515.120443(21)$ & NTT & primary & $u^{\prime} g^{\prime} i^{\prime}$ & $7.69,2.55,2.55$ & $4,5,4$ & $6.4,1.8,2.2$ \\
\hline 2010 Nov $15 \ldots$ & $55515.237910(23)$ & NTT & secondary & $u^{\prime} g^{\prime} i^{\prime}$ & $7.69,2.55,2.55$ & $4,5,3$ & $3.6,1.2,1.7$ \\
\hline 2010 Nov $25 \ldots$ & $55525.228090(18)$ & NTT & primary & $u^{\prime} g^{\prime} i^{\prime}$ & $7.69,2.55,2.55$ & $4,5,3$ & $4.2,1.5,1.6$ \\
\hline 2010 Nov $26 \ldots$ & $55526.168300(13)$ & NTT & primary & $u^{\prime} g^{\prime} r^{\prime}$ & $5.89,1.95,1.95$ & $4,5,3$ & $3.5,1.3,1.6$ \\
\hline 2010 Nov $26 \ldots$ & $55526.285767(29)$ & NTT & secondary & $u^{\prime} g^{\prime} r^{\prime}$ & $5.89,1.95,1.95$ & $4,5,2$ & $6.6,1.9,2.5$ \\
\hline 2010 Nov $27 \ldots$ & $55527.108548(12)$ & NTT & primary & $u^{\prime} g^{\prime} i^{\prime}$ & $5.89,1.95,1.95$ & $4,5,4$ & $3.4,1.1,1.5$ \\
\hline 2010 Nov $27 \ldots$ & $55527.226026(20)$ & NTT & secondary & $u^{\prime} g^{\prime} i^{\prime}$ & $5.46,1.35,1.35$ & $4,5,4$ & $3.8,1.5,1.8$ \\
\hline 2010 Nov $28 \ldots$ & $55528.166253(16)$ & NTT & secondary & $u^{\prime} g^{\prime} r^{\prime}$ & $5.89,1.95,1.95$ & $4,5,5$ & $3.3,1.1,1.3$ \\
\hline 2010 Nov $29 \ldots$ & $55529.106526(19)$ & NTT & secondary & $u^{\prime} g^{\prime} i^{\prime}$ & $5.46,1.35,1.35$ & $4,4,4$ & $4.1,1.4,2.0$ \\
\hline 2010 Dec $2 \ldots$ & $55532.162334(16)$ & NTT & secondary & $u^{\prime} g^{\prime} i^{\prime}$ & $7.69,2.55,2.55$ & $4,4,4$ & $2.6,0.9,1.3$ \\
\hline 2010 Dec $10 \ldots$ & $55540.154399(16)$ & NTT & secondary & $u^{\prime} g^{\prime} i^{\prime}$ & $7.69,2.55,2.55$ & $4,4,4$ & $2.7,0.9,1.3$ \\
\hline 2010 Dec $15 \ldots$ & $55545.208237(16)$ & NTT & primary & $u^{\prime} g^{\prime} i^{\prime}$ & $7.48,2.48,2.48$ & $4,4,4$ & $3.7,1.3,1.7$ \\
\hline 2010 Dec $16 \ldots$ & $55546.148475(18)$ & NTT & primary & $u^{\prime} g^{\prime} i^{\prime}$ & $7.48,2.48,2.48$ & $4,4,4$ & $4.7,1.6,2.0$ \\
\hline 2010 Dec $17 \ldots$ & $55547.088715(23)$ & NTT & primary & $u^{\prime} g^{\prime} i^{\prime}$ & $7.48,2.48,2.48$ & $4,4,4$ & $5.1,1.8,2.1$ \\
\hline 2010 Dec $18 \ldots$ & $55548.146459(36)$ & NTT & secondary & $u^{\prime} g^{\prime} i^{\prime}$ & $7.48,2.48,2.48$ & $4,4,4$ & $7.0,2.6,3.4$ \\
\hline 2012 Jan $17 \ldots$ & $55943.870809(09)$ & WHT & primary & $u^{\prime} g^{\prime} r^{\prime}$ & $2.48,2.48,2.48$ & $4,5,3$ & $4.1,0.7,0.8$ \\
\hline 2012 Jan $17 \ldots$ & $55943.988273(13)$ & WHT & secondary & $u^{\prime} g^{\prime} r^{\prime}$ & $4.99,2.48,2.48$ & $4,5,4$ & $2.9,0.8,0.9$ \\
\hline 2012 Jan $18 \ldots$ & $55944.928546(22)$ & WHT & secondary & $u^{\prime} g^{\prime} r^{\prime}$ & $4.99,2.48,2.48$ & $4,5,3$ & $4.1,1.2,1.5$ \\
\hline 2012 Jan $19 \ldots$ & $55945.046118(28)$ & WHT & primary & $u^{\prime} g^{\prime} r^{\prime}$ & $4.98,2.48,2.48$ & $4,5,3$ & $5.3,1.7,2.0$ \\
\hline 2012 Jan $19 \ldots$ & $55945.868766(42)$ & WHT & secondary & $u^{\prime} g^{\prime}$ & $5.99,2.98, \cdots$ & $4,5, \cdots$ & $3.7,1.3, \cdots$ \\
\hline 2012 Jan $21 \ldots$ & $55947.866825(09)$ & WHT & primary & $u^{\prime} g^{\prime} r^{\prime}$ & $3.46,1.72,1.72$ & $4,5,2$ & $2.9,0.9,1.0$ \\
\hline 2012 Jan $22 \ldots$ & $55948.924551(12)$ & WHT & secondary & $u^{\prime} g^{\prime} r^{\prime}$ & $3.99,1.98,1.98$ & $4,5,4$ & $2.9,0.8,0.9$ \\
\hline 2012 Jan $23 \ldots$ & $55949.042137(10)$ & WHT & primary & $u^{\prime} g^{\prime} r^{\prime}$ & $3.99,1.98,1.98$ & $4,5,4$ & $4.0,0.9,1.1$ \\
\hline 2012 Sep $1 \ldots$ & $56171.174286(11)$ & WHT & primary & $u^{\prime} g^{\prime} r^{\prime}$ & $5.56,1.84,1.84$ & $4,4,4$ & $3.8,1.6,1.1$ \\
\hline 2012 Sep $4 \ldots$ & $56174.230072(11)$ & WHT & primary & $u^{\prime} g^{\prime} r^{\prime}$ & $6.48,2.14,2.14$ & $5,4,4$ & $2.5,1.0,1.0$ \\
\hline 2012 Sep $10 \ldots$ & $56180.106572(12)$ & WHT & primary & $u^{\prime} g^{\prime} r^{\prime}$ & $5.00,2.48,2.48$ & $4,4,4$ & $2.8,2.1,0.9$ \\
\hline
\end{tabular}

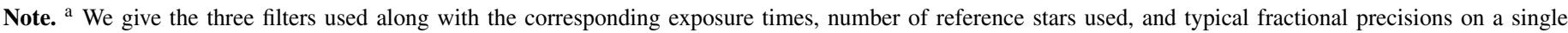
measurement of NLTT 11748.

for NLTT 11748 the original eclipse constraints from Steinfadt et al. (2010) were limited in their precision. As the system is a single-line binary, individual masses were not known. Further uncertainties came from limited photometric precision and a low observational cadence, along with ignorance of proper limb darkening for WDs of this surface gravity and temperature.

Here, we present new data and a new analysis of eclipse photometry for NLTT 11748 that rectifies almost all of the previous limitations and gives precise masses and radii that are largely model independent (at least concerning models of the ELM WDs themselves), allowing for powerful new constraints on the evolution and structure of ELM WDs. NLTT 11748 was identified by Kawka \& Vennes (2009) as a candidate ELM WD binary, containing a helium-core WD with mass $\approx 0.15 M_{\odot}$ presumably orbiting with a more typical $0.6 M_{\odot}$ carbon/oxygen (CO) WD (note that the photometric primary is the lowermass object, owing to the inverted WD mass-radius relation). While searching for pulsations, Steinfadt et al. (2010) found periodic modulation in the light curve of NLTT 11748 which they determined was due to primary $(6 \%)$ and secondary $(3 \%)$ eclipses in a $5.6 \mathrm{hr}$ orbit, as confirmed by radial velocity measurements (also see Kawka et al. 2010). The primary lowmass WD has a low surface gravity $(\log (g)=6.18 \pm 0.15$ from Kawka et al. 2010, $\log (g)=6.54 \pm 0.05$ from Kilic et al. 2010 ) and an effective temperature $T_{\text {eff }}=8580 \pm 50 \mathrm{~K}$ (Kawka et al. 2010 or $8690 \pm 140 \mathrm{~K}$ from Kilic et al. 2010). Constraints in this region of $\left(\log (g), T_{\text {eff }}\right)$ space are particularly valuable, as the behavior of systems in this region is complex with a wide range of predicted ages consistent changing over small ranges of mass, especially since it is near the transition from systems that show CNO flashes and those that do not (Althaus et al. 2013).

Our new data consist of high-cadence ( $2.5 \mathrm{~s}$ compared with $30 \mathrm{~s}$ previously), high-precision photometry in multiple simultaneous filters, which we combine with improved modeling and knowledge of limb-darkening coefficients (Gianninas et al. 2013). We outline the new observations in Section 2. The majority of the new analysis is described in Section 3, with the results in Section 3.2. Finally, we make some additional physical inferences and discuss our results in Section 4.

\section{OBSERVATIONS AND REDUCTION}

\subsection{ULTRACAM Observations}

We observed NLTT 11748 with ULTRACAM (Dhillon et al. 2007) over 27 eclipses during 2010 and 2012, as summarized in Table 1. ULTRACAM provides simultaneous fast photometry through 3 filters with negligible dead time. During 2010, it was mounted on the $3.5 \mathrm{~m}$ New Technology Telescope (NTT) at La Silla Observatory, Chile. We used the $u^{\prime}$ and $g^{\prime}$ filters, along with either $r^{\prime}$ or $i^{\prime}$. The integration times were chosen based on the conditions, but were typically $1-2 \mathrm{~s}$ for the redder filters and 5-8 s for the $u^{\prime}$ filter. During 2012, ULTRACAM was mounted on the $4.2 \mathrm{~m}$ William Herschel Telescope at the Observatorio del Roque de los Muchachos on the island of La Palma. Here, we used only the $u^{\prime} g^{\prime} r^{\prime}$ filters, although for one observation we discarded the $r^{\prime}$ data as they were corrupted. Exposure times were $2-3 \mathrm{~s}$ for the redder filters and 3-5 s for the $u^{\prime}$ filter, taking advantage of better conditions, a larger mirror, and a lower 
airmass toward this northern target. The total observing time for each eclipse was typically less than 40 minutes.

The data were reduced using custom software. We first determined bias and flatfield images appropriate for every observation. Then, we measured aperture photometry for NLTT 11748 and up to six reference sources that were typically somewhat brighter than NLTT 11748 itself. The aperture was sized according to the mean seeing for the observation, but was held fixed for a single observation and a single filter. Finally, a weighted mean of the reference star magnitudes (some of which were removed because of saturation and some of which were removed because of low signal-to-noise, especially in the $u^{\prime}$ band) was subtracted from the measurements for NLTT 11748. These detrended data were the final relative photometry that we used in all subsequent analysis. Given the short duration of the eclipse ( $\approx 3$ minutes), any remaining variations in the relative photometry due to transparency or airmass changes could be ignored; we did not attempt to model the out-of-eclipse data (cf. Shporer et al. 2010).

\subsection{Near-infrared Observations}

In addition to the ULTRACAM observations, we observed a few eclipses using the Gemini Near-Infrared Imager (NIRI; Hodapp et al. 2003) on the $8 \mathrm{~m}$ Gemini-North telescope under program GN-2010B-Q-54. Given the low signal-to-noise and relatively long cadence, the primary eclipse data were not particularly useful in constraining the properties of the system. Instead, we concentrate on the secondary eclipse observations, where the additional wavelength coverage is helpful in constraining the effective temperature of the secondary (Section 4.2). The data were taken on 2010 November 21 and 2010 December 15 using the $J$-band filter. The total duration of the observations were 37 and 31 minutes around a secondary eclipse, as predicted from our initial ephemeris. Successive exposures happened roughly every $25 \mathrm{~s}$, of which $20 \mathrm{~s}$ were actually accumulating data, so our overhead was about $20 \%$.

To reduce the data, we used the nprepare task in IRAF, which adds various meta-data to the FITS files. We then corrected the data for nonlinearities ${ }^{9}$ and applied flatfields computed using the niflat task, which compared dome-flat exposures taken with the lamp on and off to obtain the true flatfield. We used our own routines to perform point spread function photometry with a Moffat (1969) function. This function was held constant for each observation and was fit to bright reference stars. Finally, we subtracted the mean of two bright reference stars to de-trend the data.

\section{ECLIPSE FITTING}

To start, we determined rough eclipse times and shapes by fitting a simple model to the data, using a square eclipse for the secondary eclipses and a linear limb-darkening law for the primary eclipses. These results were only used as a starting point for the later analysis, but the times were correct to $\pm 10 \mathrm{~s}$. We then fit the photometry data, as summarized below. The fitting used only the ULTRACAM data; the NIRI data were added later to constrain the secondary temperature.

Our main eclipse fitting used a Markov-Chain Monte Carlo (MCMC) fitter, based on a Python implementation ${ }^{10}$ (ForemanMackey et al. 2013) of the affine-invariant ensemble sampler

\footnotetext{
9 Using the nirlin.py script from

http://staff.gemini.edu/ astephens/niri/nirlin/nirlin.py.

10 See http://dan.iel.fm/emcee/.
}

(Goodman \& Weare 2010). We parameterized the light curve according to

1. Mass and radius of the primary (low-mass) WD, $M_{1}$ and $R_{1}$

2. Orbital inclination $i$

3. Radial velocity amplitude of the primary $K_{1}$

4. Mean period $P_{B}$, reference time $t_{0}$, and time delay $\Delta t$

5. Temperatures of the primary $T_{1}$ and secondary $T_{2}$

for nine total parameters. These were further constrained by priors based on spectroscopy, with $K_{1}=273.4 \pm 0.5 \mathrm{~km} \mathrm{~s}^{-1}$ and $T_{1}=8690 \pm 140 \mathrm{~K}$ (Steinfadt et al. 2010; Kilic et al. 2010). We assumed a strictly periodic ephemeris (with no spin down; see Section 4.1) that includes a possible time delay between the primary and secondary eclipses (Kaplan 2010).

Our limb-darkening law used four-parameter (Claret 2000) limb-darkening coefficients, as determined by Gianninas et al. (2013) for a range of gravities and effective temperatures. We interpolated the limb-darkening parameters for the primary's temperature $T_{1}$, although we used several fixed values of $\log (g)$ $(6.25,6.50$, and 6.75) instead of the value for each fit. This is done both to avoid numerical difficulties in two-dimensional (2D) interpolation over a coarse grid and to avoid biasing the fitted $\log (g)$ by anything other than the light-curve shape (unlike the temperature, the different spectroscopic determinations of the surface gravity are significantly discrepant). We found that variations in $\log (g)$ used for the limb-darkening parameters changed the fit results by $<1 \sigma$.

With values for $M_{1}, i, P_{B}$, and $K_{1}$, the mass of the secondary (high-mass) WD, $M_{2}$, is then determined (and so is the mass ratio $q \equiv M_{1} / M_{2}$, as well as $K_{2}=q K_{1}$ ). The final parameter is the radius of the secondary WD $R_{2}$. However, as this is a more or less normal CO WD that is not tidally distorted $\left(\left(M_{1} / M_{2}\right)\left(R_{2} / a\right)^{3} \approx 10^{-7}\right)$, we used a mass-radius relation appropriate for WDs in this mass range (Fontaine et al. 2001; Bergeron et al. 2001), ${ }^{11}$ interpolating linearly. The complication that this introduced is that WDs with finite temperatures do have radii slightly larger than the nominal zero-temperature model and that this excess depends on the thickness of their hydrogen envelopes. For the mass and temperature range considered here, this excess is typically $r_{2} \equiv R_{2} / R_{2}\left(T_{2}=0\right)=1.02-1.06$. In what follows, we treat $r_{2}$ as a free parameter and give our results in terms of $r_{2}$, with a detailed discussion of the influence of $r_{2}$ on the other parameters in Section 3.1.

Overall, we had 22,574 photometry measurements within $\pm 400 \mathrm{~s}$ of the eclipses (shown in Figure 1), which we corrected to the solar system barycenter using a custom extension to the TEMPO2 ${ }^{12}$ pulsar timing package (Hobbs et al. 2006). The eclipses themselves were modeled with the routines of Agol (2002; also see Mandel \& Agol 2002), which accounts for intrabinary lensing (Maeder 1973; Marsh 2001).

We started 200 MCMC "walkers," where each walker executes an independent path through the parameter space. The walkers were initialized from normally distributed random variables, with each variable taken from the nominal values determined previously (Steinfadt et al. 2010; Kilic et al. 2010) with generous uncertainties. In the end, we increased the uncertainties on the initial conditions and it did not change the resulting parameter distributions. Each walker was allowed 500 iterations to "burn in," after which its memory of the sampled parameter space was deleted. Finally, each walker iterated for

\footnotetext{
11 See http://www.astro.umontreal.ca/ bergeron/CoolingModels/.

12 See http://www.atnf.csiro.au/research/pulsar/tempo2/.
} 


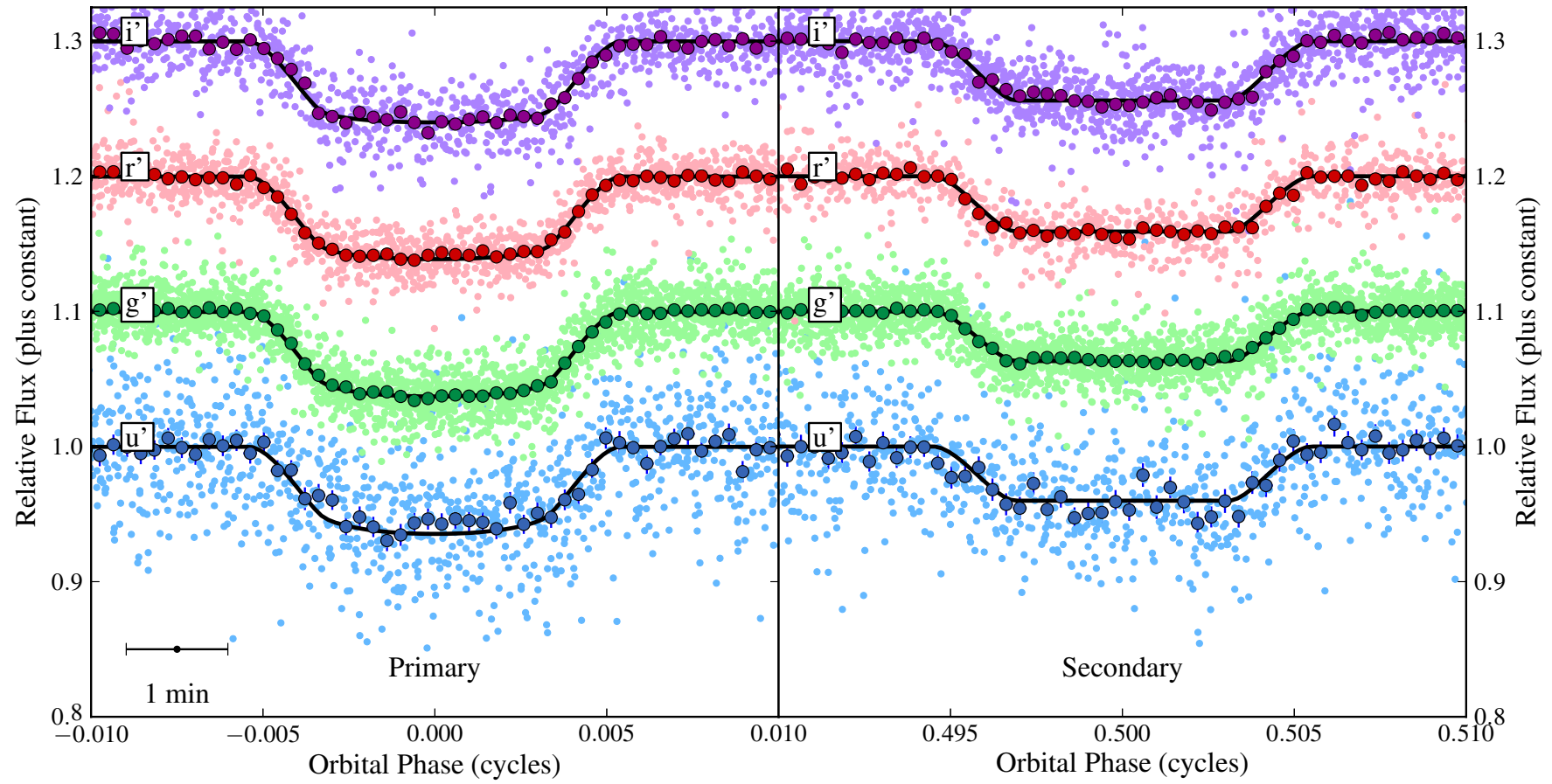

Figure 1. Normalized primary (left) and secondary (right) eclipses of NLTT 11748, as measured with ULTRACAM. The raw data are the points, while binned data are the circles with error bars and the best-fit models are the solid lines. The different filters are labeled. A 1 minute interval is indicated by the scale bar at the lower left. Data from 2010 and 2012 have been combined.

(A color version of this figure is available in the online journal.)

a further 5000 cycles, giving $200 \times 5000=1,000,000$ samples. However, not all of these are independent: we measured an auto-correlation length of about 100 samples from the resulting distributions, so we thinned the parameters by taking every 91 samples (we wanted a number near our measured autocorrelation but that was not commensurate with the number of walkers).

\subsection{The Influence of the Secondary's Envelope Thickness on the Measurement}

As discussed above, our one significant assumption (which was also made in Steinfadt et al. 2010) is that the secondary star follows the mass-radius relation for a CO WD. This seems reasonable, given inferences from observations (Kawka et al. 2010; Kilic et al. 2010) and from evolutionary theory. However, with the high precision of the current dataset, we must examine the choice of mass-radius relation closely. In particular, the zero-temperature model used in Steinfadt et al. (2010) is no longer sufficient. For effective temperatures near $7500 \mathrm{~K}$ and masses near $0.7 M_{\odot}$, finite-temperature models are larger than the zero-temperature models by roughly $2 \%$ (thin envelopes, taken to be $10^{-10}$ of the star's mass) to $6 \%$ (thick envelopes, taken to be $10^{-4}$ of the star's mass) and moreover the excess depends on mass (Fontaine et al. 2001; Bergeron et al. 2001). This excess is similar to what we observe in a limited series of models computed using Modules for Experiments in Stellar Astrophysics (Paxton et al. 2011, 2013). The envelope thickness can be constrained directly through astroseismology of pulsating WDs (ZZ Ceti stars), with most sources having thick envelope (fractional masses of $10^{-6}$ or above), but extending down such that roughly $10 \%$ of the sources have thin envelopes (fractional masses of $10^{-7}$ or below); there is a peak at thicker envelopes, but there is a broad distribution (Romero et al. 2012; consistent with the findings of Tremblay \& Bergeron 2008).

We use the parameter $r_{2}$ to explore the envelope thickness. Values near 1.02 correspond to thin envelopes (with some slight mass dependence), while values near 1.06 correspond to thick envelopes. Changing $r_{2}$ over the range of values discussed above leads to changes in the best-fit physical parameters $M_{1}, M_{2}$, $R_{1}$, and $R_{2}$. In particular, $M_{1}$ was surprisingly sensitive to $r_{2}$. Determining a "correct" value for $r_{2}$ is beyond the scope of this work, but we can understand how the physical parameters scale with $r_{2}$ in a reasonably simple manner. Since we know the period accurately and can also say that $\sin i \approx 1$ (deeply eclipsing), we know that $M_{1}+M_{2} \propto a^{3}$ (where $a$ is the semi-major axis) from Kepler's third law. We also know $K_{1}$, which is the orbital speed of the primary:

$$
K_{1} \approx \frac{2 \pi a}{P_{B}} \frac{M_{2}}{M_{1}+M_{2}}
$$

which allows us to constrain $a M_{2} \propto M_{1}+M_{2}$. Combining these gives $M_{2} \propto a^{2}$. We can further parameterize the mass-radius relation of the secondary:

$$
R_{2} \propto r_{2} M_{2}^{\beta} .
$$

The duration of the eclipse fixes $R_{1} / a$, while the duration of ingress/egress fixes $R_{2} / a$ (e.g., Winn 2011), so we can also say $a \propto R_{2}$ or $a \propto r_{2} M_{2}^{\beta}$. Combining this with $M_{2} \propto a^{2}$ gives $M_{2} \propto r_{2}^{2 /(1-2 \beta)}$. Near $M_{2} \approx 0.7 M_{\odot}$, the mass-radius relation has a slope $\beta \approx-0.78$ (compare with the traditional $\beta=-1 / 3$ for lower masses), so $M_{2} \propto r_{2}^{0.78}$. Since $a \propto M_{2}^{1 / 2}$ and both $R_{1}$ and $R_{2}$ are $\propto a, R_{1} \propto r_{2}^{1 /(1-2 \beta)} \propto r_{2}^{0.39}$ and the same for $R_{2}$ (at a fixed $M_{2}, R_{2} \propto r_{2}$, as given in Equation(2); however, the result here is for the best-fit value of $R_{2}$, which can result in changes of the other parameters including $M_{2}$ ). 


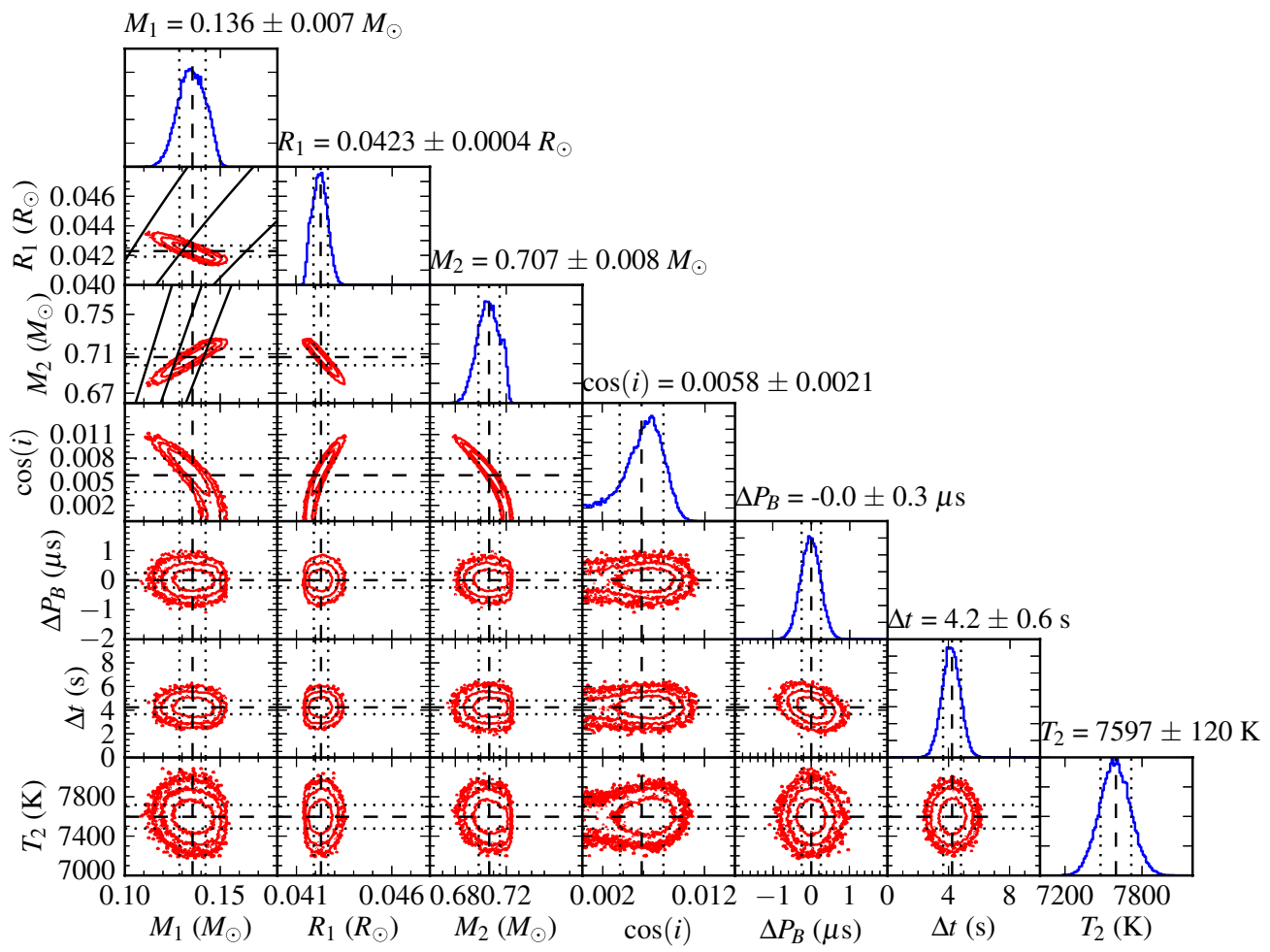

Figure 2. Joint confidence contours on the parameters from the fit of NLTT 11748, assuming $r_{2}=1.00$. We show $68 \%$, 95\%, and $99.7 \%$ contours on $2 \mathrm{D}$ distributions that have been marginalized from the $8 \mathrm{D}$ original distribution (we do not plot distributions for the reference time $t_{0}$, as it is of little physical interest). $\Delta P_{B}$ is the offset of $P_{B}$ with respect to its mean (Table 2). We also show the 1D distributions for each parameter. In all of the plots, the black dashed lines show the means and the black dotted lines show the $\pm 1 \sigma$ limits. Finally, in the plot of $M_{2}$ vs. $M_{1}$, we show solid lines corresponding to the mass ratios $q=0.16,0.18$, and 0.20 (which can map to constraints from $\Delta t$ ), while in the plot of $R_{1}$ vs. $M_{1}$, we show solid lines corresponding to $\log (g)=6.2,6.3$, and 6.4 .

(A color version of this figure is available in the online journal.)

To understand how $M_{1}$ changes with $r_{2}$, we use the Keplerian mass function, which fixes $M_{2}^{3} \propto\left(M_{1}+M_{2}\right)^{2}$. If we take the logarithmic derivative of this, we find:

$$
\frac{d \log M_{1}}{M_{1}}=\alpha \frac{d \log M_{2}}{M_{2}},
$$

with $\alpha=3 / 2+M_{2} / 2 M_{1}$. Since our mass ratio $q$ is roughly 0.2 (Table 2), we find $\alpha \approx 4.0$. From this, we find that $M_{1} \propto r_{2}^{2 \alpha /(1-2 \beta)} \propto r_{2}^{3.13}$, which is much steeper than the other dependencies. These relations are borne out by MCMC results (Table 2, Figures 2 and 3).

\subsection{Results}

The model fit the data well, with a minimum $\chi^{2}$ of 22978.8 for 22,566 degrees of freedom (dof). ${ }^{13}$ We show 1D and 2D marginalized confidence contours in Figure 2 and the best-fit light curves in Figure 1. The results are given in Table 2. A linear ephemeris gives a satisfactory fit to the data, although

\footnotetext{
${ }^{13}$ In all of our fittings, we make use of the $\chi^{2}$ statistic. This assumes that individual data points are independent of each other; on the other hand, correlated errors can become significant for very precise photometry and can alter the nature of parameter and uncertainty estimation (see Carter \& Winn 2009). To test this, we examined the out-of-eclipse data for any correlation between subsequent data points. We found autocorrelation lengths of $0-2$ samples, with a mean of 0.85 . This was very similar to the distribution of autocorrelation lengths estimated from sets of 100 uncorrelated random numbers drawn from $\mathcal{N}(1,0.003)$ (similar in length and properties to our data), so we conclude that deviations from an autocorrelation length of 0 are consistent with the finite sample sizes that we used and that the data are consistent with being independent.
}

we find a significantly non-zero value for $\Delta t$, which we discuss below.

For NIRI, the quality of the data is modest, with typical uncertainties of $\pm 0.03 \mathrm{mag}$ and a cadence of $25 \mathrm{~s}$. Given the quality of the ULTRACAM results, fitting the NIRI data with all parameters free would not add to the results. Instead, we kept the physical parameters fixed at their best-fit values from Table 2 and only fit for the eclipse depth at $1.25 \mu \mathrm{m}$.

The results are shown in Figure 4. Despite the modest quality of the data, the fit is good, with $\chi^{2}=160.7$ for 161 dof. We find a depth $d_{2}(1.25 \mu \mathrm{m})$ of $4.2 \% \pm 0.4 \%$, corresponding to a $J$-band flux ratio $d_{2}(1.25 \mu \mathrm{m}) /\left(1-d_{2}(1.25 \mu \mathrm{m})\right)$ of $4.4 \% \pm 0.4 \%$ (see Section 4.2). This value is slightly off from the predictions based on our fit to the ULTRACAM data (Figure 5), although by less than $2 \sigma$.

To derive the eclipse times in Table 1, we used the results of the full eclipse fit but fit the model (with the shape parameters held fixed) to each observation individually.

\section{DISCUSSION}

The analyses presented in Section 3 show precise determinations of the masses and radii of the WDs in the NLTT 11748 binary. We have one remaining free parameter, which is the size of the radius excess of the CO WD $r_{2}$, related to the size of its hydrogen envelope. Moreover, we have shown that the eclipse data are consistent with a linear ephemeris, although there is a systematic shift between the primary and secondary eclipses. Separately, the variation of the secondary eclipse depth with wavelength allows for accurate determination of the temperature of the CO WD, which then determines its age through 


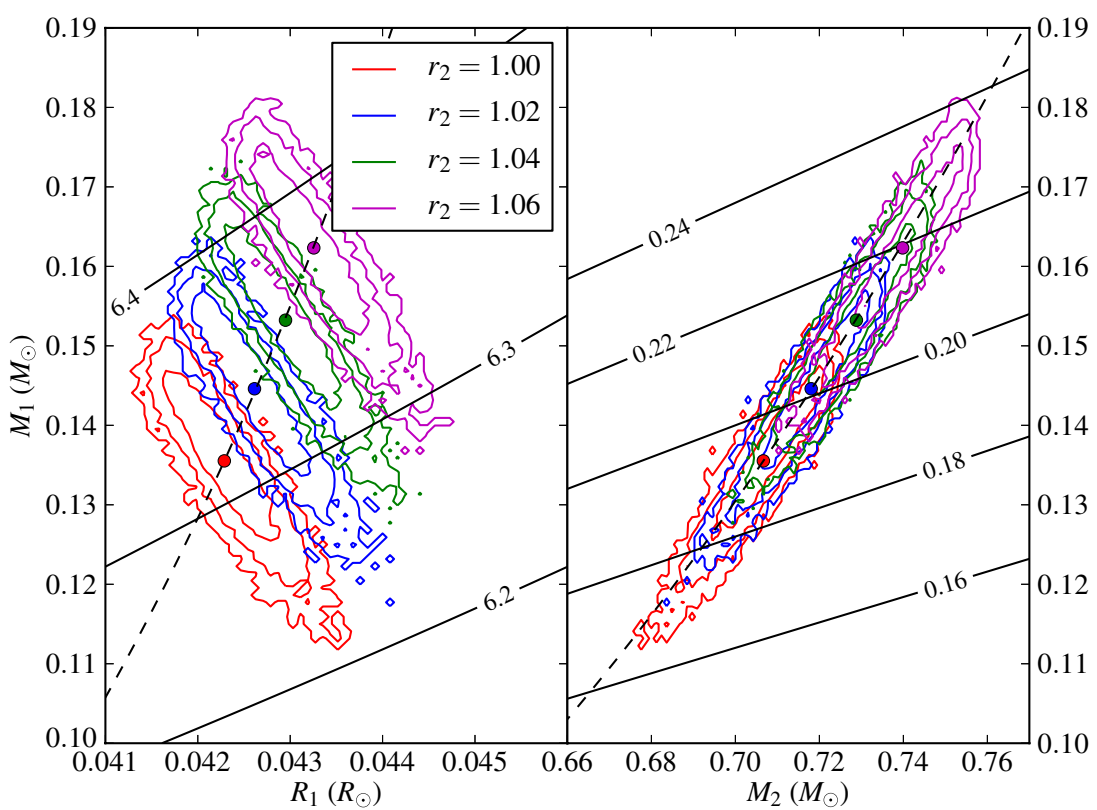

Figure 3. Mass and radius constraints as a function of $r_{2}$, showing $r_{2}=1.00$ (red), 1.02 (blue), 1.04 (green), and 1.06 (purple). In the left panel, we plot $M_{1}$ vs. $R_{1}$, while in the right panel we plot $M_{1}$ vs. $M_{2}$. In both panels, we plot the best-fit points (filled circles), along with the expected variations according to Section 3.1 (dashed lines). Additionally, we plot contours of constant surface gravity $\log (g)=6.2,6.3$, and 6.4 (left) and mass ratio $q=0.16,0.18,0.20,0.22$, and 0.24 (right) in black. (A color version of this figure is available in the online journal.)

Table 2

Eclipse Fitting Results

\begin{tabular}{|c|c|c|c|c|}
\hline Quantity & Value: $r_{2}=1.00$ & Value: $r_{2}=1.02$ & Value: $r_{2}=1.04$ & Value: $r_{2}=1.06$ \\
\hline$\overline{M_{1}{ }^{\mathrm{a}}\left(M_{\odot}\right) \ldots}$ & $0.136 \pm 0.007$ & $0.145 \pm 0.007$ & $0.153 \pm 0.007$ & $0.162 \pm 0.007$ \\
\hline$R_{1}^{\mathrm{a}}\left(R_{\odot}\right) \ldots$ & $0.0423 \pm 0.0004$ & $0.0426 \pm 0.0004$ & $0.0429 \pm 0.0004$ & $0.0433 \pm 0.0004$ \\
\hline$K_{1}^{\mathrm{a}, \mathrm{b}}\left(\mathrm{km} \mathrm{s}^{-1}\right) \ldots$ & $273.4 \pm 0.5$ & $273.4 \pm 0.5$ & $273.4 \pm 0.5$ & $273.4 \pm 0.5$ \\
\hline$R_{2}\left(R_{\odot}\right) \ldots$ & $0.0108 \pm 0.0001$ & $0.0109 \pm 0.0001$ & $0.0110 \pm 0.0001$ & $0.0111 \pm 0.0001$ \\
\hline$i^{\mathrm{a}}(\operatorname{deg}) \ldots$ & $89.67 \pm 0.12$ & $89.67 \pm 0.12$ & $89.66 \pm 0.12$ & $89.67 \pm 0.12$ \\
\hline$a\left(R_{\odot}\right) \ldots$ & $1.514 \pm 0.009$ & $1.526 \pm 0.009$ & $1.538 \pm 0.009$ & $1.549 \pm 0.009$ \\
\hline$R_{2} / R_{1} \ldots$ & $0.2565 \pm 0.0006$ & $0.2567 \pm 0.0006$ & $0.2568 \pm 0.0006$ & $0.2570 \pm 0.0006$ \\
\hline $\log (g)_{1} \ldots$ & $6.32 \pm 0.03$ & $6.34 \pm 0.03$ & $6.36 \pm 0.03$ & $6.38 \pm 0.03$ \\
\hline $\log (g)_{2} \ldots$ & $8.22 \pm 0.01$ & $8.22 \pm 0.01$ & $8.22 \pm 0.01$ & $8.22 \pm 0.01$ \\
\hline$T_{1}^{\mathrm{a}, \mathrm{b}}(\mathrm{K}) \ldots$ & $8706 \pm 136$ & $8705 \pm 137$ & $8705 \pm 135$ & $8707 \pm 136$ \\
\hline$T_{2}^{\mathrm{a}}(\mathrm{K}) \ldots$ & $7597 \pm 119$ & $7594 \pm 120$ & $7591 \pm 118$ & $7590 \pm 119$ \\
\hline$t_{0}{ }^{\mathrm{a}}(\mathrm{MBJD}) \ldots$ & $55772.041585 \pm 0.000004$ & $55772.041585 \pm 0.000004$ & $55772.041585 \pm 0.000004$ & $55772.041585 \pm 0.000004$ \\
\hline
\end{tabular}

Notes.

${ }^{a}$ Directly fit in the MCMC. All other parameters are inferred.

${ }^{\mathrm{b}}$ Used a prior distribution based on spectroscopic observations. All other prior distributions were flat.

well-studied CO WD cooling curves. Below, we discuss additional constraints on the masses, radii, and ages of the components determined by consideration of the eclipse times, secondary temperature, and distance (determined by astrometry).

\subsection{Ephemeris, Rømer Delay, and Mass Ratio Constraints}

Using the measured eclipse times from Table 1, along with those reported by Steinfadt et al. (2010), we computed a linear ephemeris for NLTT 11748 with a constant frequency $f_{B}=$ $1 / P_{B}$. The residuals (Figure 6 ) are consistent with being flat and with the results from the full eclipse fitting (Table 2), showing no indication of orbital changes. However, we do find a systematic offset between the times of the primary and secondary eclipses, as predicted in Kaplan (2010). The secondary eclipses arrive earlier on average, by $\Delta t=4.1 \pm 0.5 \mathrm{~s}$ (after correcting for this, the rms residual for the new data is $1.7 \mathrm{~s}$ and the overall $\chi^{2}$ for the ephemeris data is 38.9 with 29 dof), which is consistent with $\Delta t=4.2 \pm 0.6 \mathrm{~s}$ inferred from the full eclipse fitting (Table 2). The sign is correct for a delay caused by the light-travel delay across the system (the Rømer delay) when the more massive object is smaller, the magnitude of which is (Kaplan 2010)

$$
\Delta t_{\mathrm{LT}}=\frac{P_{B} K_{1}}{\pi c}(1-q)
$$




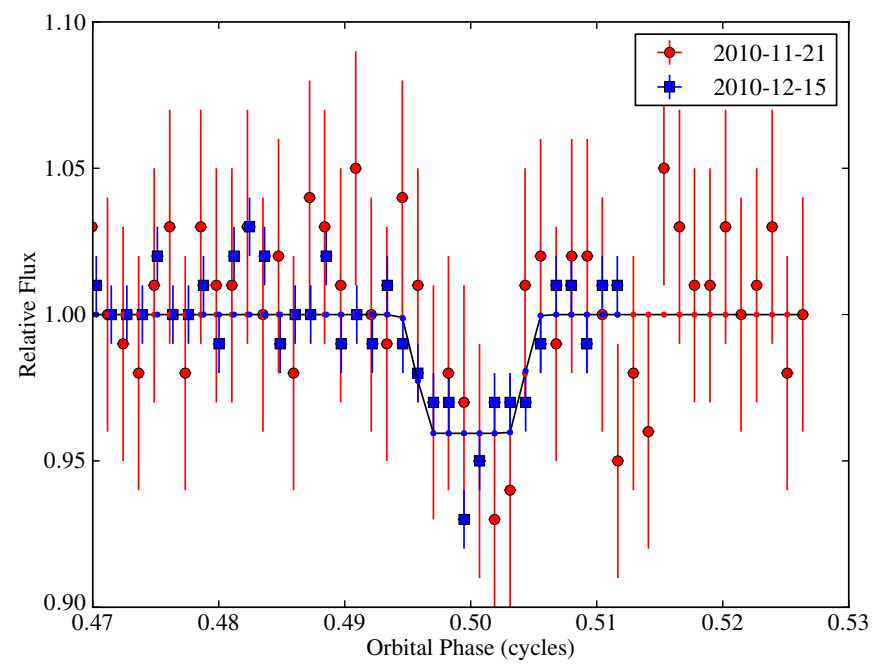

Figure 4. Secondary eclipses of NLTT 11748 observed with Gemini/NIRI. The two observations are the circles/squares, as labeled. The solid curve is the best-fit model, with the points representing the model integrated over the $20 \mathrm{~s}$ exposures for each set of observations.

(A color version of this figure is available in the online journal.)

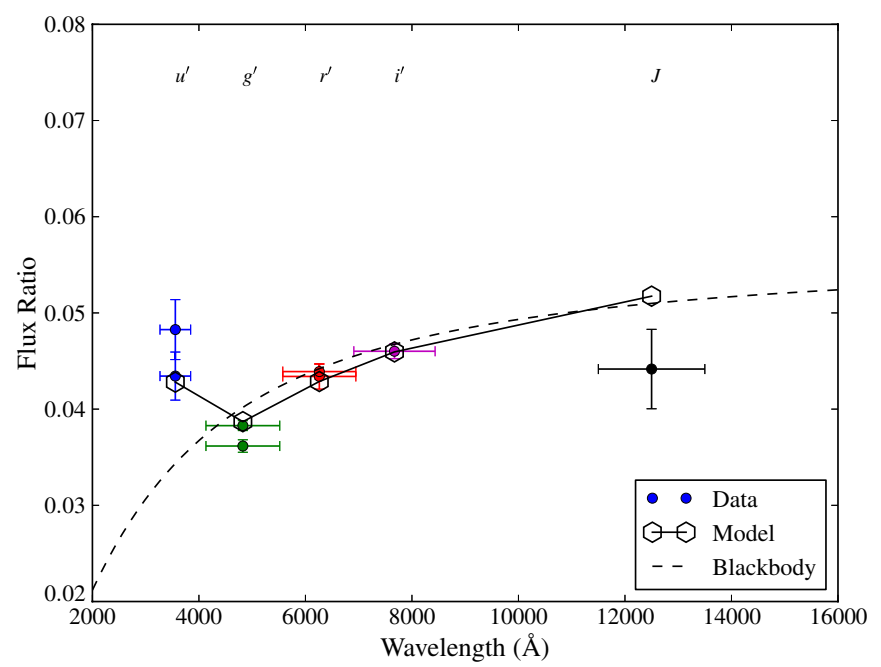

Figure 5. Secondary-to-primary flux ratio as a function of wavelength for the secondary eclipses of NLTT 11748. We plot data from 2010 and 2012 together, along with our best-fit model (open symbols). The different bands are labeled. The model is derived from Tremblay et al. (2011) synthetic photometry. We also show the corresponding flux ratio determined from blackbodies (which had been used previously), which does not match the $u^{\prime}$ data at all.

(A color version of this figure is available in the online journal.)

If we use $\Delta t=4.2 \pm 0.6 \mathrm{~s}$ (from the full eclipse fitting in Table 2), then we infer $q_{\mathrm{LT}}=0.29 \pm 0.10$. This is fully consistent with our fitted values for $q$ (Table $2 ; q=0.192 \pm 0.008$ for $\left.r_{2}=1.00\right)$. However, it is also possible that some time delay is caused by a finite eccentricity of the orbit (Kaplan 2010; Winn 2011), with

$$
\Delta t_{e}=\frac{2 P_{B} e}{\pi} \cos \omega
$$

where $e$ is the eccentricity and $\omega$ is the argument of periastron. ${ }^{14}$ However, the Rømer delay must be present in the eclipse timing with a magnitude $(4.76 \pm 0.05) r_{2}^{2.6} \mathrm{~s}$, based on our mass determination. Therefore, instead of using the Rømer

\footnotetext{
14 Note that the expression from Kaplan (2010) is missing a factor of two, as
} pointed out by Barlow et al. (2012).

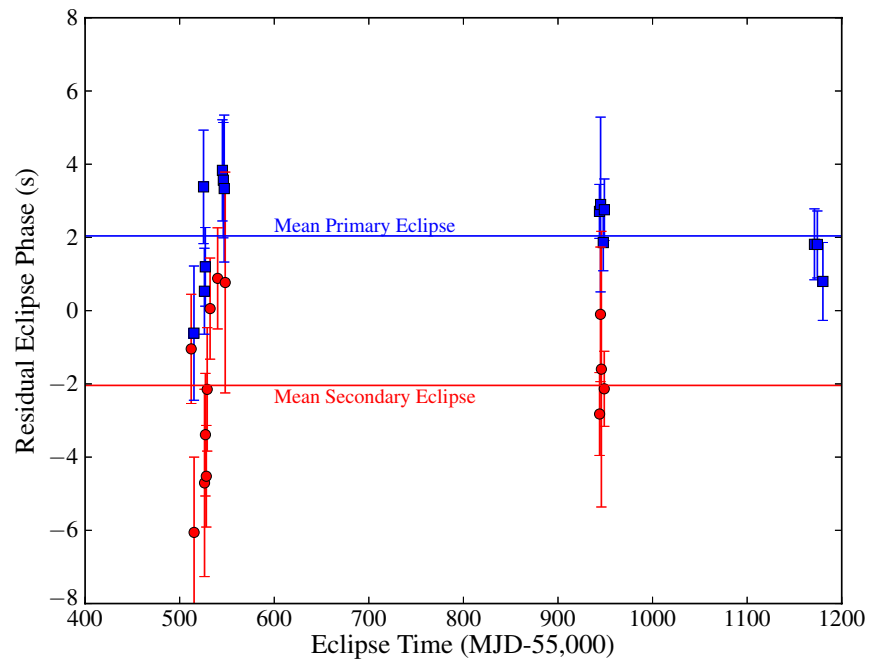

Figure 6. Residual eclipse phase vs. eclipse time of NLTT 11748, showing only the ULTRACAM measurements, although the measurements of Steinfadt et al. (2010) were included in the ephemeris calculations. The primary eclipses are the blue squares, while the secondary eclipses are the red circles. The data have been fit with a constant-frequency ephemeris. We find an offset between the mean time of the primary eclipse compared with that of the secondary eclipse of $\Delta t=4.1 \pm 0.5 \mathrm{~s}$.

(A color version of this figure is available in the online journal.)

delay to constrain the masses, we can use it to constrain the eccentricity. Doing this gives $e \cos \omega=(-4 \pm 5) \times 10^{-5}$ (consistent with a circular orbit). This would then be one of the strongest constraints on the eccentricity of any system without a pulsar, as long as the value of $\omega$ is not particularly close to $\pi / 2$ (or $3 \pi / 2$ ). This may be testable with long-term monitoring of NLTT 11748 , as relativistic apsidal precession (Blandford \& Teukolsky 1976) should be $\dot{\omega} \approx 2 \mathrm{deg} \mathrm{yr}^{-1}$ (for a nominal $e=10^{-3}$ ). As long as any tidal precession is on a longer timescale, the change in $\omega$ could separate the Rømer delay from that due to a finite eccentricity. For a system as wide as NLTT 11748 tidal effects are likely to be negligible (Fuller \& Lai 2013; Burkart et al. 2013). Further relativistic effects may be harder to disentangle: an orbital period decay $\dot{P}_{B}$ will be of a magnitude $-1 \mu \mathrm{s} \mathrm{yr}^{-1}$, compared with a period derivative from the Shklovskii (1970) effect ${ }^{15}$ of $+24 \mu \mathrm{s} \mathrm{yr}^{-1}$ (current data do not strongly constrain $\dot{P}_{B}$ because of the short baseline, with $\left.\dot{P}_{B}=(-1900 \pm 900) \mu \mathrm{s} \mathrm{yr}^{-1}\right)$. Therefore, an accurate determination of the distance (whose uncertainties currently dominate the uncertainty in the period derivative) will be necessary before any relativistic $\dot{P}_{B}$ can be measured.

In comparison, the radial velocity constraints on the eccentricity are considerably weaker: Steinfadt et al. (2010) determined $e<0.06(3 \sigma)$, while Kilic et al. (2010) and Kawka et al. (2010) both assumed circular orbits. We refit all of the available radial velocity measurements from those three papers. Assuming a circular orbit, we find $K_{1}=273.3 \pm 0.4 \mathrm{~km} \mathrm{~s}^{-1}$ (like Kilic et al. 2010, whose data dominate the fit). We also tried the eccentric orbit as parameterized by Damour \& Taylor (1991). The fit is consistent with a circular orbit, with $e \cos \omega=0.001 \pm 0.004$ and $e \sin \omega=-0.004 \pm 0.004$, which limits $e<0.017(3 \sigma)$. The eclipse durations can also weakly constrain the eccentricity, with the ratio of the secondary eclipse to the primary eclipse

\footnotetext{
15 The Shklovskii (1970) $\dot{P}_{B}$ (also known as secular acceleration) is particularly large in the case of NLTT 11748 because of its proximity and large space velocity (Kawka \& Vennes 2009). If measured, it can be used to derive a
} geometric distance constraint (Bell \& Bailes 1996). 
duration roughly given by $1+2 e \sin \omega$ (for $e \ll 1$ ), although as noted by Winn (2011) this is typically less useful than the constraints on $e \cos \omega$ from eclipse timing. In the future, we can fit for this term directly.

While all data are satisfactorily fit by just a simple linear ephemeris, we can also ask if a third body could be present in the system. Such a body, especially if on an inclined orbit, could significantly speed up the merger of the inner binary and may alter the evolution of the system (Thompson 2011). Any putative tertiary would likely be in a more distant circumbinary orbit, since the interactions necessary to produce the ELM WD would have disrupted closer companions. A tertiary would produce transit timing variations (Holman \& Murray 2005; Agol et al. 2005), moving the eclipse times we measure. A full analysis of transit timing variations, including nonlinear orbital interactions, is beyond the scope of this paper. Instead, we did a limited analysis where we considered the system to be sufficiently hierarchical such that the inner binary was unperturbed (consistent with our measurements) and only its center of mass moved due to the presence of the tertiary. We took the ephemeris residuals and fit a variety of periodic models, determining for each trial period what the maximum amplitude could be (marginalizing over phase) such that $\chi^{2}$ increased by 1 from the linear ephemeris fit (adding additional terms in general decreases $\chi^{2}$, but we wanted to see what the maximum possible amplitude could be). We found that for periods of 1-300 days, the limit on any sinusoidal component was $\lesssim 1$ s (consistent with the rms discussed above) or smaller than the orbit of the inner binary. Therefore, unless it is highly inclined, no tertiary with such a period is possible. As we get to periods that are longer than 300 days, we no longer have sufficient data to constrain a periodic signal, but here the constraints from our polynomial fit also exclude any stellar-mass companion (an amplitude of $1 \mathrm{~s}$ at a period of 300 days would require a mass of $0.002 M_{\odot}$ if the outer orbit is also edge-on).

\subsection{Secondary Temperature and Age Constraints}

As with the NIRI data, we can separate the fitting of the secondary eclipse depth from the rest of the eclipse fitting and derive the eclipse depths as a function of wavelength, $d_{2}(\lambda)$ (where we also separate the 2010 and 2012 ULTRACAM observations). Given the eight secondary eclipse depths that we measure, we can determine the ratio of the radius of the secondary to the primary $R_{2} / R_{1}$ as well as the temperature of the secondary, $T_{2}$, given measurements of $T_{1}$. For that, we use the determination by Kilic et al. (2010) $-T_{1}=8690 \pm$ $140 \mathrm{~K}$-largely consistent with the value determined by Kawka et al. (2010) of $8580 \pm 50 \mathrm{~K}$. The secondary eclipse depths are related to the wavelength-dependent flux ratios:

$$
f(\lambda) \equiv \frac{F_{2}(\lambda)}{F_{1}(\lambda)}=\frac{d_{2}(\lambda)}{1-d_{2}(\lambda)}=\frac{R_{2}^{2} 10^{-m\left(\lambda, T_{2}\right) / 2.5}}{R_{1}^{2} 10^{-m\left(\lambda, T_{1}\right) / 2.5}},
$$

where $m(\lambda, T)$ is the absolute magnitude of a fiducial WD with a temperature $T$ at wavelength $\lambda$.

To determine the secondary's temperature from the eclipse depths, we first sample $T_{1}$ from the distribution $\mathcal{N}(8690,140)$ 1000 times. Then, for each sample, we interpolate the synthetic photometry of Tremblay et al. (2011; using the $0.2 M_{\odot}$ model) to determine the photometry for the primary, $m\left(\lambda, T_{1}\right)$. We then solve for the temperature of the secondary and the radius ratio by minimizing the $\chi^{2}$ statistic, comparing our measured $f(\lambda)$ with the synthetic values (using the $0.7 M_{\odot}$ grid for the secondary; the results did not change if we used the $0.6 M_{\odot}$ or $0.8 M_{\odot}$ grids instead).

The resulting distribution of $T_{2}$ and $\left(R_{2} / R_{1}\right)$ is independent of any assumptions in the global eclipse fitting. We find $T_{2}=7643 \pm 94 \mathrm{~K}$ and $R_{2} / R_{1}=0.255 \pm 0.002$. However, the fits had an average $\chi^{2}=12.6$ for 5 dof, mostly coming from a small mismatch between the inferred eclipse depth at $g^{\prime}$ measured in 2010 versus 2012. If we increase the uncertainties to have a reduced $\chi^{2}=1$, then we find $T_{2}=7643 \pm 150 \mathrm{~K}$ and $R_{2} / R_{1}=0.255 \pm 0.003$. Note that the radius ratio here is fully consistent with that inferred from the fit to the rest of the eclipse shape (Section 3 and Table 2).

We show the results in Figure 5 (including the results of the NIRI data analysis), along with the results using blackbodies for the flux distributions instead of the synthetic photometry (as had been done by Steinfadt et al. 2010 and others). It is apparent that the blackbody does not agree well and using the synthetic photometry is vital.

The constraints using the separate wavelength-dependent eclipse depths ended up being slightly less precise than the value from the full eclipse fitting, although the two constraints are entirely consistent. We therefore choose the values from Table 2, where we determined a secondary temperature of $T_{2}=7600 \pm 120 \mathrm{~K}$. Using the thin (thick) hydrogen atmosphere models for a $0.7 M_{\odot} \mathrm{CO} \mathrm{WD}$, we find a secondary age $\tau_{2}=1.70 \pm 0.09$ Gyr $(1.58 \pm 0.07 \mathrm{Gyr})$ by interpolating the cooling curves from Tremblay et al. (2011). So, the envelope uncertainty does not contribute significantly to the uncertainty in the age of the secondary directly. A bigger contribution is through changes in the secondary mass $M_{2}$.

\subsection{Primary Radius and Distance Constraints}

Based on the measured $J$-band photometry $(J=$ $15.84 \pm 0.08$ from Skrutskie et al. 2006) ${ }^{16}$, along with an estimate for the extinction $\left(E(B-V)=0.10\right.$ and $R_{V}=3.2$, from Kilic et al. 2010), we can compare our measured radius with that inferred from a parallax measurement $(\pi=5.6 \pm 0.9$ mas; H. Harris 2011, private communication). We again use the Tremblay et al. (2011) synthetic photometry for the bolometric correction at the temperature determined by Kilic et al. (2010) and use $A_{J}=0.29 A_{V}$. Based on these data, we infer $R_{1, \text { phot }}=0.049 \pm 0.009 R_{\odot}$. This is fully consistent (within $0.5 \sigma$ ) with our inferred values from the eclipse fitting. We can use this value along with the radius ratio inferred from the eclipse shape (roughly $R_{2} / R_{1}=0.2567 \pm 0.0006$ ) to determine $R_{2}=0.013 \pm 0.002 R_{\odot}$. From here, we can calculate $M_{2, \text { thin }}=0.57 \pm 0.08 M_{\odot}$ and $M_{2 \text {,thick }}=0.61 \pm 0.11 M_{\odot}$, which makes use of evolutionary models that were interpolated to the correct effective temperature (Fontaine et al. 2001; Bergeron et al. 2001). These masses are a little lower than our secondary masses calculated by the eclipse fitting, but differ by less than $2 \sigma$. Inverting the problem, we infer based on our eclipse fitting for $r_{2}=1.02$ a distance $d=159 \pm 8$ pc $(\pi=6.3 \pm 0.3$ mas $)$, with the uncertainty dominated by the uncertainty in the photometry.

Our analysis above included the effects of in-binary microlensing. Steinfadt et al. (2010) assumed microlensing would modify the primary eclipse depth, but did not show definitively that it was required for a good fit (cf. Muirhead et al. 2013).

\footnotetext{
16 Kawka \& Vennes (2009) incorrectly give the 2MASS $J$-band magnitude as $15.873 \pm 0.077$, but the online database lists 2MASS J03451680+1748091 as having $J=15.837 \pm 0.077$.
} 
We fit the same data with the same procedure as in Section 3, but did not allow for any decrease in the depth of the primary eclipse from lensing. The resulting fit was adequate, although slightly worse than with lensing. With no lensing amplification, to match the depth of the primary eclipse requires a reduced value of $R_{2} / R_{1}$ or a lower inclination. We can do that by increasing $R_{1}$ or decreasing $R_{2}$, but that is difficult as the eclipse durations fix $R_{1} / a$ and $R_{2} / a$. We end up accomplishing this by increasing the masses, which widens the orbit (increasing $a$ to go along with the increase in $R_{1}$ and decreasing $R_{2}$ through the mass-radius relation for the WD). For $r_{2}=1.00$, we find $M_{1}=0.16 M_{\odot}$ and $M_{2}=0.74 M_{\odot}$, along with $R_{1}=0.044 R_{\odot}$ $\left(\log (g)_{1}=6.36 \pm 0.04\right)$. While this combination of parameters does not give as good a fit to the data as the fit with lensing, the difference is not statistically significant, with an increase in $\chi^{2}$ of 20 (the reduced $\chi^{2}$ increased from 1.018 to 1.019 , for a chance of occurrence of about $50 \%$ ). We would need other independent information (such as a much more precise parallax or a more precise time delay) to break the degeneracies.

\section{CONCLUSIONS}

Using extremely high-quality photometry combined with improved modeling, we have determined the masses and radii of the WDs in the NLTT 11748 binary to better than $\pm 0.01 M_{\odot}$ and $\pm 0.0005 R_{\odot}$ statistical precision, although uncertainties in the radius excess limit our final precision. This analysis makes use of the eclipse depth and shape, including corrections for gravitational lensing, and is consistent with the Rømer delay measured independently from eclipse times, $\Delta t=4.2 \pm 0.6 \mathrm{~s}$. This would be the first detection of an observed Rømer delay for ground-based eclipse measurements (cf. Bloemen et al. 2012; Barlow et al. 2012), although in all of these systems there is the possibility that the time delay is instead related to a finite, but small, eccentricity.

Our mass measurement for the smallest plausible radius excess $\left(r_{2}=1.02\right), M_{1}=0.137 \pm 0.007 M_{\odot}$, is significantly lower than that inferred by Kilic et al. (2010) on the basis of the Panei et al. (2007) evolutionary models or that inferred by Althaus et al. (2013) from newer models. Even for the highest value of $r_{2}$ that we considered (1.06), we still find a primary mass of $0.157 \pm 0.008 M_{\odot}$, significantly below the $0.17-0.18 M_{\odot}$ range discussed by Kilic et al. (2010) and Althaus et al. (2013). This may call for a revision of those models to take into account the improved observational constraints or it may indicate that an even higher value of $r_{2}$ is more realistic. In any case, our surface gravity is lower than that from Kilic et al. (2010), which was used by Althaus et al. (2013), while it is consistent to within $1 \sigma$ with the gravity measured by Kawka et al. (2010). With our $\log (g)$ determination, the mass is closer to the prediction from Althaus et al. (2013, who find $M=0.174 M_{\odot}$ for $\log (g)=6.40$ and $\log _{10} T_{\text {eff }}=3.93$, compared with $0.183 M_{\odot}$ for the higher $\log (g))$, although again high values of $r_{2}$ are required. However, our cooling age for the secondary is a factor of two to three smaller than the cooling age of $>4$ Gyr for the primary predicted by the Althaus et al. (2013) models for the lower mass. This might be a reflection of the non-monotonic evolution experienced by some ELM WDs, even though NLTT 11748 seems to have a low enough mass that it would cool in a simpler manner. Further constraints on the mass ratio from eclipse timing or direct detection of the secondary's spectrum (the inferred values of $K_{2}$ in Table 2 vary significantly) could help resolve this question.
We thank the anonymous referee for helpful comments. This work was supported by the National Science Foundation under grants PHY 11-25915 and AST 11-09174. T.R.M. was supported under a grant from the UK's Science and Technology Facilities Council (STFC), ST/F002599/1. V.S.D., S.P.L., and ULTRACAM were supported by the STFC. A.N.W. was supported by the University of Wisconsin, Milwaukee Office of Undergraduate Research. We thank the staff of Gemini for assisting in planning and executing these demanding observations. We thank D. Foreman-Mackey for help using emcee and L. Althaus for supplying evolutionary models. Based on observations obtained at the Gemini Observatory, which is operated by the Association of Universities for Research in Astronomy, Inc., under a cooperative agreement with the NSF on behalf of the Gemini partnership: the National Science Foundation (United States); the National Research Council (Canada); CONICYT (Chile); the Australian Research Council (Australia); Ministério da Ciência, Tecnologia, e Inovação (Brazil); and Ministerio de Ciencia, Tecnología, e Innovación Productiva (Argentina).

Facilities: Gemini:Gillett (NIRI), NTT (ULTRACAM), ING:Herschel (ULTRACAM)

\section{REFERENCES}

Agol, E. 2002, ApJ, 579, 430

Agol, E., Steffen, J., Sari, R., \& Clarkson, W. 2005, MNRAS, 359, 567

Althaus, L. G., Miller Bertolami, M. M., \& Córsico, A. H. 2013, A\&A, 557, A19

Antoniadis, J., van Kerkwijk, M. H., Koester, D., et al. 2012, MNRAS, 423, 3316

Barlow, B. N., Wade, R. A., \& Liss, S. E. 2012, ApJ, 753, 101

Bassa, C. G., van Kerkwijk, M. H., Koester, D., \& Verbunt, F. 2006, A\&A, 456,295

Bell, J. F., \& Bailes, M. 1996, ApJL, 456, L33

Bergeron, P., Leggett, S. K., \& Ruiz, M. T. 2001, ApJS, 133, 413

Bergeron, P., Saffer, R. A., \& Liebert, J. 1992, ApJ, 394, 228

Blandford, R., \& Teukolsky, S. A. 1976, ApJ, 205, 580

Bloemen, S., Marsh, T. R., Degroote, P., et al. 2012, MNRAS, 422, 2600

Brown, W. R., Kilic, M., Allende Prieto, C., Gianninas, A., \& Kenyon, S. J. 2013, ApJ, 769, 66

Brown, W. R., Kilic, M., Hermes, J. J., et al. 2011, ApJL, 737, L23

Burkart, J., Quataert, E., Arras, P., \& Weinberg, N. N. 2013, MNRAS, 433, 332

Carter, J. A., \& Winn, J. N. 2009, ApJ, 704, 51

Claret, A. 2000, A\&A, 363, 1081

Damour, T., \& Taylor, J. H. 1991, ApJ, 366, 501

D’Antona, F., Ventura, P., Burderi, L., \& Teodorescu, A. 2006, ApJ, 653,1429

Deloye, C. J., Bildsten, L., \& Nelemans, G. 2005, ApJ, 624, 934

Dhillon, V. S., Marsh, T. R., Stevenson, M. J., et al. 2007, MNRAS, 378, 825

Fontaine, G., Brassard, P., \& Bergeron, P. 2001, PASP, 113, 409

Foreman-Mackey, D., Hogg, D. W., Lang, D., \& Goodman, J. 2013, PASP, 125,306

Fuller, J., \& Lai, D. 2013, MNRAS, 430, 274

Gianninas, A., Strickland, B. D., Kilic, M., \& Bergeron, P. 2013, ApJ, 766, 3 Goodman, J., \& Weare, J. 2010, Comm. App. Math. Comp. Sci., 5, 65

Hobbs, G. B., Edwards, R. T., \& Manchester, R. N. 2006, MNRAS, 369, 655

Hodapp, K. W., Jensen, J. B., Irwin, E. M., et al. 2003, PASP, 115, 1388

Holman, M. J., \& Murray, N. W. 2005, Sci, 307, 1288

Iben, I., Jr., \& Tutukov, A. V. 1984, ApJS, 54, 335

Kaplan, D. L. 2010, ApJL, 717, L108

Kaplan, D. L., Bhalerao, V. B., van Kerkwijk, M. H., et al. 2013, ApJ, 765,158

Kaplan, D. L., Bildsten, L., \& Steinfadt, J. D. R. 2012, ApJ, 758, 64

Kawka, A., \& Vennes, S. 2009, A\&A, 506, L25

Kawka, A., Vennes, S., \& Vaccaro, T. R. 2010, A\&A, 516, L7

Kilic, M., Allende Prieto, C., Brown, W. R., et al. 2010, ApJL, 721, L158

Kilic, M., Brown, W. R., Allende Prieto, C., et al. 2012, ApJ, 751, 141

Lorimer, D. R., Lyne, A. G., Festin, L., \& Nicastro, L. 1995, Natur, 376, 393

Maeder, A. 1973, A\&A, 26, 215

Mandel, K., \& Agol, E. 2002, ApJL, 580, L171

Marsh, T. R. 2001, MNRAS, 324, 547 
Marsh, T. R., Dhillon, V. S., \& Duck, S. R. 1995, MNRAS, 275, 828

Marsh, T. R., Nelemans, G., \& Steeghs, D. 2004, MNRAS, 350, 113

Moffat, A. F. J. 1969, A\&A, 3, 455

Muirhead, P. S., Vanderburg, A., Shporer, A., et al. 2013, ApJ, 767, 111

Panei, J. A., Althaus, L. G., Chen, X., \& Han, Z. 2007, MNRAS, 382, 779

Parsons, S. G., Marsh, T. R., Gänsicke, B. T., Drake, A. J., \& Koester, D. 2011, ApJL, 735, L30

Paxton, B., Bildsten, L., Dotter, A., et al. 2011, ApJS, 192, 3

Paxton, B., Cantiello, M., Arras, P., et al. 2013, ApJS, 208, 4

Romero, A. D., Córsico, A. H., Althaus, L. G., et al. 2012, MNRAS, 420, 1462

Serenelli, A. M., Althaus, L. G., Rohrmann, R. D., \& Benvenuto, O. G. 2002, MNRAS, 337, 1091

Shklovskii, I. S. 1970, SvA, 13, 562

Shporer, A., Kaplan, D. L., Steinfadt, J. D. R., et al. 2010, ApJL, 725, L200
Skrutskie, M. F., Cutri, R. M., Stiening, R., et al. 2006, AJ, 131, 1163

Steinfadt, J. D. R., Kaplan, D. L., Shporer, A., Bildsten, L., \& Howell, S. B. 2010, ApJL, 716, L146

Tauris, T. M., Langer, N., \& Kramer, M. 2012, MNRAS, 425, 1601

Thompson, T. A. 2011, ApJ, 741, 82

Tremblay, P.-E., \& Bergeron, P. 2008, ApJ, 672, 1144

Tremblay, P.-E., Bergeron, P., \& Gianninas, A. 2011, ApJ, 730, 128

van Kerkwijk, M. H., Bassa, C. G., Jacoby, B. A., \& Jonker, P. G. 2005, in ASP Conf. Ser. 328, Binary Radio Pulsars, ed. F. A. Rasio \& I. H. Stairs (San Francisco, CA: ASP), 357

Vennes, S., Thorstensen, J. R., Kawka, A., et al. 2011, ApJL, 737, L16

Webbink, R. F. 1984, ApJ, 277, 355

Winn, J. N. 2011, in Exoplanet Transits and Occultations, ed. S. Piper (Tucson, AZ: Univ. Arizona Press), 55 